\title{
Building the pediatric radiology department of the future
}

\author{
Raymond Sze
}

(C) Springer-Verlag 2011

Building the pediatric radiology of the future will involve building physical environments that optimize patient care and experience; it will require building clinical operations that enable evidence-based, safe and multidisciplinary care; and it will require building services around emerging technologies that will make pediatric diagnosis and treatment more precise and less invasive.

\section{Physical environments}

Healthcare environments are frequently cold and unwelcoming; diagnostic imaging equipment is usually intimidating to children and their families; staff environments often make it harder, rather than easier, to take care of patients. The Pediatric Radiology Department of the Future will incorporate research and insights in healthcare architecture and design to improve the family experience and optimize ergonomics and workflow to enhance the ability of the professional staff to take care of its patients.

\section{Wonder}

Careful thought to room design and colors and to softening or masking the appearance of imaging equipment can potentially convert a harrowing experience to more of a ride or experience filled with interest and even wonder and delight. At its most sophisticated and engaging, a room can

Disclaimer Dr. Sze has no financial interests, investigational or off-label uses to disclose.

\section{R. Sze $(\bowtie)$}

Children's National Medical Center,

Washington, DC 20010, USA

e-mail: rsze@cnmc.org be designed around a theme or narrative, enabling the staff to engage the child in a story, transforming the experience from fearful to fun, and ideally also decreasing reliance on sedation for studies requiring the child to hold still for extended periods of time. One radiology scan room designer remarked that he knew he had succeeded when, following her imaging study, a child turned to her parents and asked: "Can I come back?"

\section{Ease}

Careful attention to way-finding and signage helps reduce family stress and the incidence of late and lost patients. Intuitive, graphic, VISIBLE, and multilingual signage helps families navigate hospital labyrinths, especially those patients who have multiple studies within the department, or who come from or follow up with clinics throughout the institution. Distances families need to walk to and within the department should be as short and clearly marked as possible. For the staff, a careful appreciation for workflow and adjacent location of rooms and equipment can minimize the sheer number of miles and time spent walking. Many strategies and workstation solutions have emerged for creating more ergonomic environments for radiologists, cutting down on eye strain and repetitive-stress fatigue and enhancing productivity and comfort.

\section{Access}

The Pediatric Radiology Department of the Future will make it simple for referring physicians and other providers to access scheduling, results and pediatric radiologists for consultation. Reading rooms will incorporate design features that welcome the referring physician or team for consultation and discussion but also preserve privacy and 
concentration for the radiologist. In an era where PACS and remote viewing discourage busy physicians from traveling to the radiology department, it is more important than ever to maintain person-to-person contact in the interests of clear communication and accurate patient care. Concerns that "we never see our clinical colleagues" can be addressed by radiologists journeying out of the safety of their reading rooms into patient care environments, conducting imaging rounds and teaching conferences on the floors.

\section{Clinical operations}

Clinical operations and procedures are frequently arbitrary and cumbersome; patient safety remains a significant challenge; turf battles and concerns about eroding professional domain create anxiety over the future of radiology and radiologists. The Pediatric Radiology Department of the Future will incorporate simplified and standardized workflow and evidence-based protocols enabled by userfriendly informatics; it will embrace and incorporate the principles of the high-reliability organization; and it will recognize that imaging is ultimately larger than any one specialty or turf and arriving at the best diagnosis and care for the patient requires collaboration between radiologists with strong clinical knowledge and clinicians with strong imaging knowledge.

\section{Informatics}

Decision rules and appropriateness criteria will be integrated into physician ordering systems in order to have point-of-care impact on imaging test utilization. Ordering physicians will be able to see what tests have already been performed, preventing unnecessary duplication. Robust and ongoing education of referring physicians and patients is also a requisite to minimize confusion and misunderstanding regarding the rationale behind strategies for imaging test selection. Emerging systems to provide easy access to patient medical information and pathology will pull radiologists out of the clinical information and follow-up vacuum they frequently face when interpreting studies, enabling more accurate initial diagnoses and the ability to learn from interpretive errors.

\section{High-reliability organization}

The double-edged sword of radiological exams includes the risk of the physical probe, such as ionizing radiation and cataract and cancer risk; the environment, such as the powerful magnetic field that can make missiles of ferromagnetic objects; and various intravenous and oral contrast agents, which can cause immediate allergic reaction or delayed and debilitating systemic affects. Interpretive and communication errors can have equally devastating consequences. Morbidity and mortality from medical error remain a pervasive challenge and lessons or principles of the high-reliability organization provide an important organizational framework. These principles include: (1) preoccupation with failure of all sizes and shapes; (2) reluctance to simplify interpretations; (3) sensitivity to operations; (4) commitment to resilience; and (5) tapping into expertise at all levels of the organization.

\section{Collaborative biomedical imaging}

In the Pediatric Radiology Department of the Future, turf battles will be overcome by radiologists assuming a leadership role in image quality, performance improvement, safety, evidence-based technology utilization, and imaging research and by taking an inclusive approach to nonradiology clinicians interested in imaging diagnosis and therapy. The best imaging diagnoses and best outcomes for the patients will combine the expertise in imaging technology and diagnoses of the radiologist with the clinical knowledge and applied science understanding of the clinician imager.

\section{Emerging technologies and services}

Despite the remarkable advances in imaging technology, resolution and speed, imaging interpretation remains largely subjective and descriptive; many surgical procedures could be significantly improved and even replaced with image guidance and image-guided therapies; and most imaging technology and use of contrast agents are confined to detecting anatomical or structural abnormalities. Emerging quantitative, biological, and molecular-based imaging technologies will find clinical application in the Pediatric Radiology Department of the Future, dramatically enhancing the role of radiologists and enabling more precise and reproducible diagnoses and assessment of treatment response, less invasive image-guided therapies, and biologically targeted theragnostics.

\section{Phenomics}

The remarkable and inexorable advances in image resolution and speed will be matched by computer-assisted morphometric, comparison and correlative tools to help the radiologist process the overwhelming hundreds and even thousands of images per study. Subjective interpretations will be augmented with quantitative descriptors. These tools must either be automated or highly intuitive or their application will be limited. Reproducible and quantitative metrics of anatomical and pathological form will enable true genotype-phenotype correlation, improved 
and standardized pre-procedural planning, and more accurate and reliable post-therapeutic assessment.

Image-guided therapy

Image guidance and fusion will integrate seamlessly with many surgical procedures and technologies, augmenting laparoscopic and robotic surgeries and enabling surgeons to see beneath the surface with "X-ray vision" and to benefit from GPS-like navigation and procedural planning. The role and domain of interventional radiology will expand dramatically and some surgical procedures will be refined or even replaced by image-guided assistance or alternatives. Interventional radiologists will become tightly knit with their surgical colleagues and services and the field might ultimately evolve into a fused discipline of image-guided therapy, blending the two disciplines and offering more than one pathway toward expertise.

Molecular imaging

Molecular imaging is ultimately concerned with understanding and imaging the biological basis of disease, as opposed to the anatomical or physiological parameters detected and described by the majority of current imaging technology. Theragnostic nanoparticle and radiotracer probes will enable non-invasive biopsies of neoplasms and will not be limited by sampling error; nanoparticle constructs will enable targeting and delivery of chemo- or bio-therapeutic payloads to neoplasms and minimize collateral damage to surrounding normal tissue; and imaging markers of biological phenomena such as neoangiogenesis or apoptosis will enable earlier detection and quantification of therapeutic response.

\section{Conclusion}

The Pediatric Radiology Department of the Future will be planned and built around the needs of children and their families and with a keen understanding of the needs of the pediatric imaging professionals taking care of them; it will provide the most precise and least invasive care, in the safest and most cost-effective manner, and in an environment that provides clarity, warmth and, ideally, a sense of wonder. 